\title{
Machine-learning-based exchange correlation functional with physical asymptotic constraints
}

\author{
Ryo Nagai $\odot,{ }^{1,2, *}$ Ryosuke Akashi, ${ }^{2}$ and Osamu Sugino ${ }^{1,2}$ \\ ${ }^{1}$ The Institute for Solid State Physics, The University of Tokyo, Kashiwa, Chiba 277-8581, Japan \\ ${ }^{2}$ Department of Physics, The University of Tokyo, Hongo, Bunkyo-ku, Tokyo 113-0033, Japan
}

(Received 30 November 2021; accepted 2 February 2022; published 11 February 2022)

\begin{abstract}
Density functional theory is the standard theory for computing the electronic structure of materials, which is based on a functional that maps the electron density to the energy. However, a rigorous form of the functional is not known and has been heuristically constructed by interpolating asymptotic constraints known for extreme situations, such as isolated atoms and uniform electron gas. Recent studies have demonstrated that the functional can be effectively approximated using machine learning (ML) approaches. However, most ML models do not satisfy asymptotic constraints. In this paper, by applying a ML model architecture, we demonstrate a neural network-based exchange-correlation functional satisfying physical asymptotic constraints. Calculations reveal that the trained functional is applicable to various materials with an accuracy higher than that of existing functionals, even for materials whose electronic properties are not included in training dataset. Our proposed method thus improves the accuracy and generalization performance of the ML-based functional by combining the advantages of ML and analytical modeling.
\end{abstract}

DOI: 10.1103/PhysRevResearch.4.013106

\section{INTRODUCTION}

Density functional theory (DFT) [1] is a method for electronic structure calculation. It is useful for elucidating the physical properties of various materials and plays an important role in industrial applications, such as drug discovery and semiconductor development. In principle, the electronic structure can be obtained by wave function theory (WFT), which directly solves the Schrödinger equation. However, the computational cost increases exponentially with respect to the number of electrons, which prohibits computation for materials with tens of atoms or more. Alternatively, the electronic structure can be obtained by solving the Kohn-Sham (KS) equation in DFT with a cubic computational cost [2]. This makes it possible to compute the electronic structure of materials with up to millions of atoms. As a result, DFT has become for calculating the electronic structure.

However, at the cost of the reduced computational complexity, the KS equation includes a term whose rigorous form is unknown. This term is called the exchange-correlation (XC) functional, which relates the electron density to the manybody interaction of electrons. This term has been heuristically approximated in various ways. The most common form of the $\mathrm{XC}$ functional is a combination of several asymptotic limits formulated with physical theories, such as the uniform electron gas limit [3]. It has been argued that satisfying as

\footnotetext{
*r-nag@issp.u-tokyo.ac.jp

Published by the American Physical Society under the terms of the Creative Commons Attribution 4.0 International license. Further distribution of this work must maintain attribution to the author(s) and the published article's title, journal citation, and DOI.
}

many physical constraints as possible is important to increase the performance of the approximation [4]. However, the combination of physical constraints is often performed with analytically tractable models, which may be insufficient to represent the intrinsic complexity of the XC functional.

In this context, another approach has emerged, namely, the application of machine learning (ML). By training a flexible ML model with high-quality material data, one can construct $\mathrm{XC}$ functionals containing more complex electronic interactions than by the analytical modeling. This method has been demonstrated to yield successful results in various systems. [5-7] Brockherde et al. proposed a ML mapping to obtain electronic structures with lower computational cost than by the KS equation [6]. This method was demonstrated to be effective in accelerating materials simulations; however, it can only be applied to materials composed of the same chemical elements as those used in training. Nagai et al. applied ML to improve the accuracy of the XC functional in the KS equation $[7,8]$. Their ML models are designed to be applicable to materials containing any chemical element; however, its performance becomes unreliable for materials that differ significantly from the materials in the training dataset. In some cases, the convergence of the calculation becomes unstable.

These problems can be solved by including all archetypal materials with all chemical elements in the training dataset; however, this is impractical because of the difficulty of obtaining such a large amount of data. Because it is difficult to obtain electronic states with sufficient accuracy via experiments, training data are mainly generated by calculation. To exceed the accuracy of conventional DFT, the data should be generated using WFT because its accuracy must be higher than that of DFT with the approximated XC functional. However, the computational complexity of modern WFT is $\mathcal{O}\left(N^{7}\right)$ 
or higher, where $N$ denotes the system size. Thus, the materials available for training are limited to small molecules. The greatest challenge in the ML-based modeling is to achieve high generalization performance with limited data.

As a solution to this problem, a method of imposing physical constraints on ML functionals has been proposed. Hollingsworth et al. imposed a physical constraint on the MLbased noninteracting kinetic energy functional in a 1D model system [9]. Without the physical constraint, the constructed kinetic energy functional could take any possible electron density as its input and predict the corresponding energy even if it did not resemble the training data. Alternatively, by imposing a coordinate-scaling condition on the density, Hollingsworth et al. limited the target of ML to density with a specific spatial width. By transforming the output of the ML model with the scaling condition, they obtained the kinetic energy applicable to electron densities with various system widths. Their results demonstrated that ML models with the coordinatescaling condition learned the system with higher accuracy than ML models without constraints. This method reduced the burden on ML by compressing the dimensionality of the target system. Therefore, by introducing physical constraints appropriately, the accuracy and generalization performance of ML models can be improved.

As an extension of this approach, we propose a method to construct a ML-based XC functional for real threedimensional materials satisfying physical asymptotic constraints. Several asymptotic behaviors of the XC functional in 3D systems have been derived analytically, which various approximate functionals have been developed to satisfy. The importance of physical constraints in improving transferability has been argued [4]. We believe that even in the construction of a ML XC functional, applying appropriate physical constraints regularize the behavior of the functional when there is insufficient training.

In this paper, we propose a novel architecture to allow a ML model to satisfy the asymptotic constraints. We apply this architecture to $\mathrm{XC}$ functional construction based on a neural network (NN), and present training and benchmarking of the NN-based XC functional that satisfies as many physical asymptotic constraints as possible.

\section{METHODOLOGY}

\section{A. Imposing asymptotic constraints on ML models}

Suppose that we want a ML model $f$ to satisfy an asymptotic constraint $f(\mathbf{x}) \rightarrow f_{0}$ at $\mathbf{x} \rightarrow \mathbf{x}_{0}$. One approach is to make the training data include values at that asymptotic limit. However, ML models trained in this way do not always take the exact asymptotic value, unless the training data is strictly fitted. Alternatively, we propose making ML model satisfy the constraint in an analytical way. Instead of training the output of the ML function $f$ directly, we train the following processed form:

$$
\hat{\theta}_{\left\{\mathbf{x}_{0}, f_{0}\right\}}[f](\mathbf{x})=f(\mathbf{x})-f\left(\mathbf{x}_{0}\right)+f_{0} .
$$

Here, this operation is represented by $\hat{\theta}_{\left\{\mathbf{x}_{0}, f_{0}\right\}}$. The same ML model is substituted for the first and second terms on the right-hand side. This operator forces the model to satisfy the constraint $\hat{\theta}_{\left\{\mathbf{x}_{0}, f_{0}\right\}}[f](\mathbf{x})=f_{0}$ strictly.

Furthermore, suppose that we have $N_{c}$ constraints, where $f$ converges to $f_{0}^{(1)}, f_{0}^{(2)}, \ldots f_{0}^{\left(N_{c}\right)}$ at $\mathbf{x}_{0}^{(1)}, \mathbf{x}_{0}^{(2)}, \ldots, \mathbf{x}_{0}^{\left(N_{c}\right)}$. We propose the following formula, which, constructed as a generalization of the Lagrange interpolation, satisfies all the constraints:

$$
\hat{\Theta}_{i=1, \ldots N_{c}}[f](\mathbf{x})=\sum_{i=1}^{N_{c}} \prod_{j \neq i} \frac{l_{i, j}\left(\mathbf{x}-\mathbf{x}_{j}\right)}{l_{i, j}\left(\mathbf{x}_{i}-\mathbf{x}_{j}\right)} \hat{\theta}_{\left\{x_{0}^{(i)}, f_{0}^{(i)}\right.}[f](\mathbf{x}) .
$$

Here, we represent this operation by $\Theta . l_{i, j}\left(i, j=1,2, \ldots N_{c}\right)$ are function satisfying $l_{i, j}(\mathbf{x})=0$ at $|\mathbf{x}|=0$. Although further tuning can be performed, we set $l_{i, j}(x)=\tanh \left(|\mathbf{x}|^{2}\right)$ for all $\{i, j\}$ in this mark. It converges to 1 when $|\mathbf{x}|$ is large, and thus it suppresses the oscillations coming from the high dimension polynomial in Eq. (2). Depending on the form of $l_{i, j}$, the behavior around each asymptotic limit differs, but we expect it is not significant as the difference can be absorbed by the flexible ML part $f$.

\section{B. KS equation in DFT}

Here we demonstrate the effectiveness of this architecture by applying it to the problem of constructing an XC functional. Based on DFT, the electronic structure of materials can be calculated by solving the following $\mathrm{KS}$ equations:

$$
\begin{gathered}
\left\{-\frac{\nabla^{2}}{2}+V_{\text {ion }}(\mathbf{r})+V_{\mathrm{H}}(\mathbf{r})+V_{\mathrm{XC}}(\mathbf{r})\right\} \varphi_{i}(\mathbf{r})=\epsilon_{i} \varphi_{i}(\mathbf{r}), \\
V_{\mathrm{H}}(\mathbf{r})=\int d \mathbf{r}^{\prime} \frac{n\left(\mathbf{r}^{\prime}\right)}{\left|\mathbf{r}-\mathbf{r}^{\prime}\right|}, \\
n(\mathbf{r})=\sum_{i=1}^{N_{\text {occ }}}\left|\varphi_{i}(\mathbf{r})\right|^{2} .
\end{gathered}
$$

Equation (3) is an eigenvalue equation, where the $i$ th eigenfunction and eigenvalue are represented by $\varphi_{i}$ and $\epsilon_{i}$, respectively. $N_{\text {occ }}$ represents the number of occupied orbitals of the material, while $V_{\text {ion }}$ is the Coulomb potential, which depends on the atomic position of each material. The forms of $V_{\mathrm{H}}$ and $V_{\mathrm{XC}}$ depend on the density $n$. The solution of this equation yields $n$; however, at the same time, $n$ is necessary for the construction of the equation itself. Therefore, the equation should be solved self-consistently; that is, the equation is solved iteratively, updating the terms using $n$ obtained in the previous step, until $n$ converges numerically. After solving the $\mathrm{KS}$ equation, the total energy $E$ can be obtained as follows:

$$
\begin{aligned}
E= & \sum_{i=1}^{N_{\mathrm{occ}}} \epsilon_{i}-\frac{1}{2} \int d \mathbf{r} \int d \mathbf{r}^{\prime} \frac{n(\mathbf{r}) n\left(\mathbf{r}^{\prime}\right)}{\left|\mathbf{r}-\mathbf{r}^{\prime}\right|} \\
& -\int d \mathbf{r} n(\mathbf{r}) V_{\mathrm{XC}}(\mathbf{r})+E_{\mathrm{XC}}[n] .
\end{aligned}
$$

$E_{\mathrm{XC}}$ and $V_{\mathrm{XC}}$ are called $\mathrm{XC}$ functionals, and follow the relation below:

$$
V_{\mathrm{XC}}(\mathbf{r})=\frac{\delta E_{\mathrm{XC}}[n]}{\delta n(\mathbf{r})} .
$$

The rigorous form of the $\mathrm{XC}$ functional as an explicit functional of $n$ is unknown. In practical use, the functional 


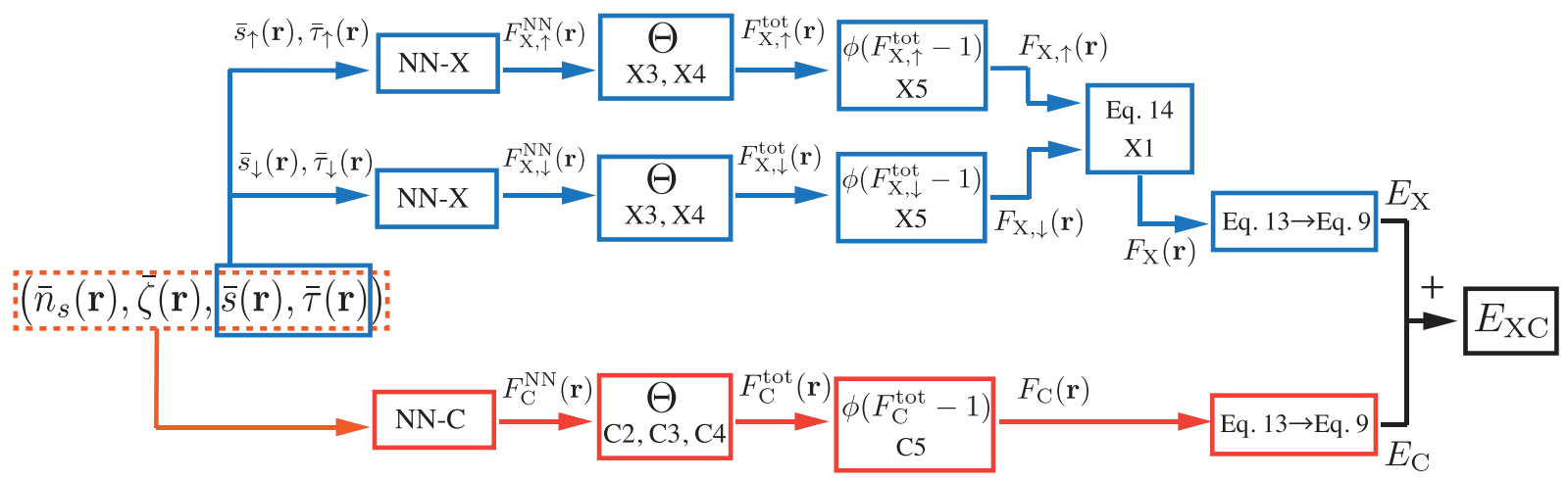

FIG. 1. Schematic diagram of the complete architecture of the physically constrained neural network XC functional. $\Theta$ represents the operation to apply the asymptotic constraints presented in Eqs. (1) and (2), while $\phi$ represents the activation function expressed in Eq. (A1).

is approximated in various ways [3,10-14], and the accuracy of the DFT calculation depends on the quality of this approximation.

\section{Interpreting the $\mathrm{XC}$ functional as a ML model}

Here, we describe the method for constructing the MLbased $\mathrm{XC}$ functional. First, we divide the $\mathrm{XC}$ functional into exchange (X) and correlation (C) parts:

$$
E_{\mathrm{XC}}[n]=E_{\mathrm{X}}[n]+E_{\mathrm{C}}[n] .
$$

Instead of taking the total $\mathrm{X}$ and $\mathrm{C}$ energy as the approximation target, we approximate the $\mathrm{XC}$ energy density $\varepsilon_{\mathrm{XC}}$ :

$$
E_{\mathrm{X}, \mathrm{C}}[n]=\int d \mathbf{r} n(\mathbf{r}) \varepsilon_{\mathrm{X}, \mathrm{C}}[n](\mathbf{r}) .
$$

Subscript "X,C" indicates the equation applies to the $\mathrm{X}$ and $\mathrm{C}$ parts, respectively. By properly constructing the local form $\varepsilon_{\mathrm{XC}}$, the functional can be applied to systems of any size. Furthermore, for the convenience of calculation, most conventional studies have adopted the semilocal approximation, where $\varepsilon_{\mathrm{X}, \mathrm{C}}[n](\mathbf{r})$ depends locally on the electron density around $\mathbf{r}$. In this study, we adopt the a meta-generalized gradient approximation (meta-GGA), where $\varepsilon_{X, C}[n](\mathbf{r})$ refers to the following local density features [12]:

$$
\begin{aligned}
n_{s}(\mathbf{r}) & =n(\mathbf{r})^{\frac{1}{3}}, \\
\zeta(\mathbf{r}) & =\frac{n_{\uparrow}(\mathbf{r})-n_{\downarrow}(\mathbf{r})}{n(\mathbf{r})}, \\
s(\mathbf{r}) & =\frac{|\nabla n(\mathbf{r})|}{n(\mathbf{r})^{\frac{4}{3}}}, \\
\tau(\mathbf{r}) & =\frac{1}{2} \sum_{i=1}^{N_{\text {occ }}}\left|\nabla \varphi_{i}(\mathbf{r})\right|^{2},
\end{aligned}
$$

where $n_{\uparrow}$ and $n_{\downarrow}$ represent the density of electrons with each spin, and $n=n_{\uparrow}+n_{\downarrow}$. Furthermore, in this paper, each feature value is processed as follows:

$$
\begin{aligned}
& \bar{n}_{s}(\mathbf{r})=\tanh \left(n_{s}(\mathbf{r})\right), \\
& \bar{\zeta}(\mathbf{r})=\tanh \left(\frac{1}{2}\left\{(1+\zeta(\mathbf{r}))^{\frac{4}{3}}+(1-\zeta(\mathbf{r}))^{\frac{4}{3}}\right\}\right),
\end{aligned}
$$

$$
\begin{aligned}
& \bar{s}(\mathbf{r})=\tanh (s(\mathbf{r})), \\
& \bar{\tau}(\mathbf{r})=\tanh \left(\frac{\tau(\mathbf{r})-\tau_{\text {unif }}(\mathbf{r})}{\tau_{\text {unif }}(\mathbf{r})}\right),
\end{aligned}
$$

where $\tau_{\text {unif }}$ is the value that $\tau$ converges to the uniform electron gas limit:

$$
\tau_{\text {unif }}(\mathbf{r})=\frac{3}{10}\left(3 \pi^{2}\right)^{\frac{2}{3}} n(\mathbf{r})^{\frac{5}{3}} .
$$

"tanh" conversions are used for the following reasons. First, it standardizes the features by arranging their variable range to be the same. Second, it allows us to deal numerically with asymptotic constraints at infinity. The limit $x \rightarrow \infty$ is converted to the finite $\operatorname{limit} \tanh (x) \rightarrow 1$.

Instead of training the entire XC functional, we designed a ML model as the factor from an existing analytical functional, the strongly constrained and appropriately normed (SCAN) functional [13]:

$$
\varepsilon_{\mathrm{X}, \mathrm{C}}[n]=\varepsilon_{\mathrm{X}, \mathrm{C}}^{\mathrm{SCAN}}[n] F_{\mathrm{X}, \mathrm{C}}[n]
$$

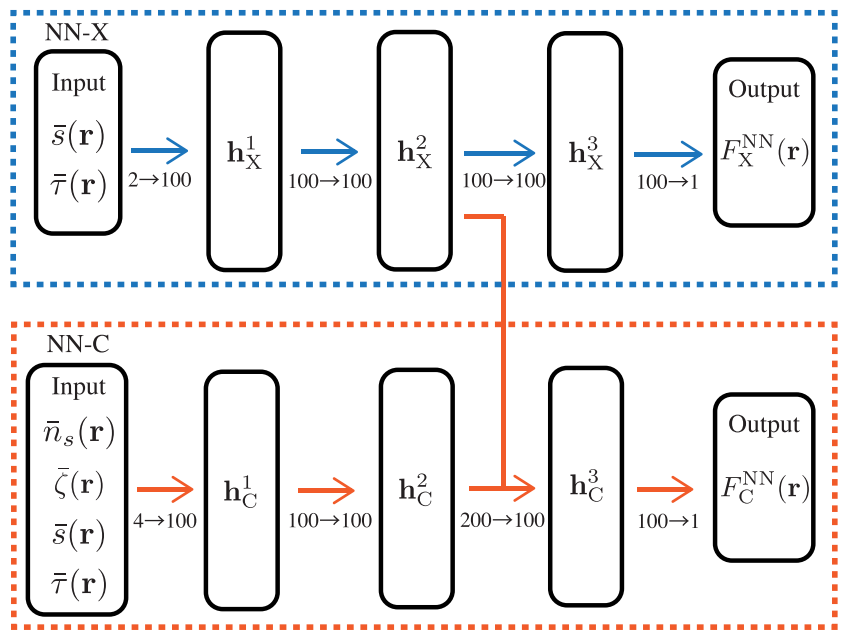

FIG. 2. Schematic diagram of the neural-network architecture. The middle layers $\mathbf{h}_{\mathrm{X}}^{2}$ and $\mathbf{h}_{\mathrm{C}}^{2}$ are concatenated to compute $\mathbf{h}_{\mathrm{C}}^{3}$. The numbers between the layers represent the dimension of the matrices connecting the layers. 
TABLE I. Applied physical constraints. Numbers starting with X denote the constraints applied to the exchange part, while those starting with $\mathrm{C}$ denote the constraints applied to the correlation part. Items in column $\mathbf{x}_{\mathbf{0}}$ correspond to the input of meta-generalized gradient approximation models: $(\bar{s}, \bar{\tau})$ for X, and $\left(\bar{n}_{s}, \bar{\zeta}, \bar{s}, \bar{\tau}\right)$ for $\mathrm{C}$ [see Eqs. (15) and (16)]. Column $f_{0}$ presents the asymptotic limits that $F_{\mathrm{X}, \mathrm{C}}$ should converge to.

\begin{tabular}{llll}
\hline \hline & \multicolumn{1}{c}{ Physical constraint } & $\mathbf{x}_{\mathbf{0}}$ & \multicolumn{1}{c}{$f_{0}$} \\
\hline $\mathrm{X} 1$ & Correct uniform coordinate density-scaling behavior & & [imposed by Eqs. (14) and (15)] \\
$\mathrm{X} 2$ & Exact spin scaling relation & $(0,0)$ & 1 \\
$\mathrm{X} 3$ & Uniform electron gas limit & $(1, \bar{\tau})$ & 1 \\
$\mathrm{X} 4$ & $F_{\mathrm{X} \text { vanishes as } s^{-1 / 2} \text { at } s \rightarrow \infty}$ & $\left(\bar{n}_{s}, \bar{\zeta}, 0,0\right)$ & 1 \\
$\mathrm{X} 5$ & Negativity of $\varepsilon_{\mathrm{X}}$ & $(0, \bar{\zeta}, \bar{s}, \bar{\tau})$ & $F_{\mathrm{C}}^{\mathrm{NN}}\left(\bar{n}_{s}, 0, \bar{s}, \bar{\tau}\right)-F_{\mathrm{C}}^{\mathrm{NN}}(0,0, \bar{s}, \bar{\tau})+1$ \\
$\mathrm{C} 1$ & Uniform electron gas limit & $(1, \bar{\zeta}, \bar{s}, \bar{\tau})$ & 1 \\
$\mathrm{C} 2$ & Uniform density scaling to low-density limit & & 1 \\
$\mathrm{C} 3$ & Weak dependence on $\zeta$ in low-density region & & \\
$\mathrm{C} 4$ & Uniform density scaling to high-density limit & & \\
$\mathrm{C} 5$ & Nonpositivity of $\varepsilon_{\mathrm{C}}$ & \\
\hline \hline
\end{tabular}

SCAN satisfies the largest number of physical constraints among existing meta-GGA approximations and is one of the most popular analytical XC functionals due to its accuracy. Using a well-constructed analytical functional can help in pretraining.

According to the correct uniform coordinate densityscaling condition [15], $\varepsilon_{\mathrm{X}}$ is independent of $\bar{n}_{s}$. Furthermore, by the exact spin scaling relation [16], the spin dependence of $\mathrm{X}$ energy satisfies the following relation:

$$
E_{\mathrm{X}}\left[n_{\uparrow}, n_{\downarrow}\right]=\frac{1}{2}\left(E_{\mathrm{X}}\left[2 n_{\uparrow}\right]+E_{\mathrm{X}}\left[2 n_{\downarrow}\right]\right) .
$$

For these constraints, we simply need to construct $F_{\mathrm{X}}$ as a function that depends only on $\bar{s}$ and $\bar{\tau}$ :

$$
F_{\mathrm{X}}[n](\mathbf{r})=F_{\mathrm{X}}(\bar{s}(\mathbf{r}), \bar{\tau}(\mathbf{r})) .
$$

In contrast, the $\mathrm{C}$ part depends on all four features:

$$
F_{\mathrm{C}}[n](\mathbf{r})=F_{\mathrm{C}}\left(\bar{n}_{s}(\mathbf{r}), \bar{\zeta}(\mathbf{r}), \bar{s}(\mathbf{r}), \bar{\tau}(\mathbf{r})\right) .
$$

In this study, we implemented $F_{\mathrm{X}, \mathrm{C}}$ using NNs. Here, we note that the differentiation in Eq. (7) can be implemented using automatic differentiation NN libraries. We used JAX [17] to implement the NN.

\section{Imposing physical constraints on NN-based XC functional}

Here, we demonstrate how to construct a physically constrained NN (pcNN)-based XC functional. Figures 1 and 2 present the schematic diagrams of the architecture. For both the $\mathrm{X}$ and $\mathrm{C}$ parts, we used a fully connected $\mathrm{NN}$ with three hidden layers, with each layer containing 100 nodes. We referred to the optimal NN size reported by Ref. [8]. The middle layer of the NN of the X part was combined with that of the $\mathrm{C}$ part to efficiently share possible common characteristics between them.

For the constructed NNs $F_{\mathrm{X}}^{\mathrm{NN}}$ and $F_{\mathrm{C}}^{\mathrm{NN}}$, we imposed the asymptotic constraints using the operation $\Theta$. The physical constraints applied in this study are listed in Table I. Constraints X3 [3] and X4 [18] were applied for the X part, while constraints C1 [10], C2 [13], C3 [12,19], and C4 [20] were applied for the $\mathrm{C}$ part. Since the XC energy density of SCAN in Eq. (13) satisfies constraints $\mathrm{X} 3, \mathrm{X} 4, \mathrm{C} 1, \mathrm{C} 2$, and $\mathrm{C} 4$, the total
$\varepsilon_{\mathrm{XC}}$ can satisfy them by converging to $f_{0}=1$. In $f_{0}$ of the constraint $\mathrm{C} 3$, the output of the NN with $\bar{\zeta}=0\left(F_{\mathrm{C}}^{\mathrm{NN}}\left(\bar{n}_{s}, 0, \bar{s}, \bar{\tau}\right)\right)$ is used to suppress the $\zeta$ dependence. By canceling the first and second terms, $f_{0}$ converges to 1 at the low-density limit, and thus satisfies constraint $\mathrm{C} 2$. Although we adopted SCAN as the base $\mathrm{XC}$ functional to impose the constraints simply, same asymptotic constraints can be imposed even if the base $\mathrm{XC}$ functional is not SCAN. In that case, the $f_{0}$ should be set as "(the value at which the asymptotic constraint converges in that limit)/(the value at which the base $\mathrm{XC}$ functional converges in that limit)". The computational cost of this pcNN functional is higher than that of analytical $\mathrm{XC}$ functionals as the computation of $\mathrm{NN}$ part requires additional $\mathcal{O}(N)$ time. However, it is not significant in total because computational cost of diagonalization of KS equation is dominant [approximately $\mathcal{O}\left(N^{3}\right)$ ]. For small materials, the pcNN is obviously slower than the analytical XC functionals, while the difference becomes trivial for larger materials.

\section{E. Loss functions}

The loss function consists of the atomization energy (AE), the ionization potential (IP), and electron densities of $\mathrm{H}_{2} \mathrm{O}$, $\mathrm{NH}_{3}$, and $\mathrm{CH}_{2}$ molecules:

$$
\begin{aligned}
\Delta= & c_{1}\left(\Delta_{\mathrm{AE}}^{\mathrm{H}_{2} \mathrm{O}}+\Delta_{\mathrm{AE}}^{\mathrm{NH}_{3}}+\Delta_{\mathrm{AE}}^{\mathrm{CH}_{2}}\right) / \mathrm{AE}_{0} \\
& +c_{2}\left(\Delta_{\mathrm{IP}}^{\mathrm{H}_{2} \mathrm{O}}+\Delta_{\mathrm{IP}}^{\mathrm{NH}_{3}}+\Delta_{\mathrm{IP}}^{\mathrm{CH}_{2}}\right) / \mathrm{IP}_{0} \\
& +c_{3}\left(\Delta_{n}^{\mathrm{H}_{2} \mathrm{O}}+\Delta_{n}^{\mathrm{NH}_{3}}+\Delta_{n}^{\mathrm{CH}_{2}}\right),
\end{aligned}
$$

TABLE II. Benchmark results for atomization energies of 144 molecules. (MAE, mean absolute error; ME, mean error; SD, standard deviation of signed error.)

\begin{tabular}{lccc}
\hline \hline XC & $\begin{array}{c}\text { MAE } \\
(\mathrm{kcal} / \mathrm{mol})\end{array}$ & $\begin{array}{c}\mathrm{ME} \\
(\mathrm{kcal} / \mathrm{mol})\end{array}$ & $\begin{array}{c}\mathrm{SD} \\
(\mathrm{kcal} / \mathrm{mol})\end{array}$ \\
\hline PBE & 17.3 & 16.2 & 13.1 \\
SCAN & 6.2 & -4.5 & 5.7 \\
NN-based & 4.8 & 1.8 & 6.3 \\
pcNN-based & 3.6 & 0.3 & 4.5 \\
\hline \hline
\end{tabular}


TABLE III. Benchmark results for lattice constants of 48 solids. Parentheses in the "NN-based" row indicate that the numerical calculations for six solids did not converge; thus, only the converged calculations were used for the statistics.

\begin{tabular}{lccc}
\hline \hline XC & $\begin{array}{c}\text { MAE } \\
(\mathrm{m} \AA)\end{array}$ & $\begin{array}{c}\text { ME } \\
(\mathrm{m} \AA)\end{array}$ & $\begin{array}{c}\mathrm{SD} \\
(\mathrm{m} \AA)\end{array}$ \\
\hline PBE & 38.1 & 33.9 & 44.2 \\
SCAN & 22.3 & -7.5 & 28.5 \\
NN-based & $(22.9)$ & $(0.8)$ & $(32.0)$ \\
pcNN-based & 19.1 & -2.5 & 26.5 \\
\hline \hline
\end{tabular}

where $\Delta_{\mathrm{AE}}, \Delta_{\mathrm{IP}}$, and $\Delta_{n}$ represent errors between the calculated and reference values of the AE, IP, and density, respectively (see Appendix D for dependence of learning efficiency on training dataset). Both $\mathrm{AE}_{0}$ and $\mathrm{IP}_{0}$ were set to 1 hartree. In this paper, the coefficients are set to $c_{1}=1$, $c_{2}=1$, and $c_{3}=10$ to make the average magnitudes of all the terms similar. The reference atomization energies, ionization energies, and electron densities were obtained by the coupled-cluster singles and doubles plus perturbative triples [CCSD(T)] method [21]. The inclusion of the electron density, a continuous quantity in 3D space, provided a large amount of information for training the weights of the NN. The error of the electron density was defined as follows:

$$
\Delta_{n}=\frac{1}{N_{e}} \sqrt{\int d \mathbf{r}\left(n^{\mathrm{DFT}}(\mathbf{r})-n^{\mathrm{CCSD}(\mathrm{T})}(\mathbf{r})\right)^{2}},
$$

where $N_{e}$ represents the number of electrons of the target material. We used PySCF [22] to perform the DFT calculation to compute the energies and densities. We also used numerical integration in Eq. (18). The number of numerical grids was on average 30000 points per molecule. Using this loss function, we trained the pcNN. See Appendix B for the detailed training procedures. The trained NN parameters and codes to call the XC functional are available on GitHub [23] (PySCF programs to use and train the pcNN), [24] (VASP add-on), and [25].

\section{F. Benchmark settings}

We evaluated the performance of the trained $\mathrm{pcNN}$ by using it as an $\mathrm{XC}$ functional in the $\mathrm{KS}$ equation to investigate the accuracy of the electronic structure of various materials.
As the test dataset, we referred to the AEs of 144 molecules, which were the same as those reported in the Training section in Ref. [26]. The DFT calculations were performed using PySCF. Additionally, because the performance for solid-state materials is also important, we conducted a test for the lattice constants of 48 solids including insulators, semiconductors, and metals, provided by [27]. The calculations for solids were performed using the Vienna Ab-initio Simulation Package (VASP) [28,29], which is commonly used software program for calculating periodic systems. We used the pseudopotentials supplied by VASP, which are based on a projector augmented wave method and adjusted on the Perdew-BurkeErnzerhof (PBE) functional [11,30,31]. For the convergence of the KS cycle, the commutator direct inversion of the iterative subspace method [32] and the Pulay mixing method [33] with default parameter settings were used in PySCF and VASP respectively. The list of materials and pseudopotentials used for each element are provided in the Supplemental Material [34].

\section{RESULTS AND DISCUSSION}

\section{A. Performances for molecules and solids}

Table II presents the benchmark results for molecules. We compared the performance of the $\mathrm{XC}$ functional constructed in this paper, denoted as "pcNN-based", with the performance of the XC functional constructed using a simple NN without satisfying any asymptotic constraints, denoted as "NN-based" [8]. Additionally, PBE and SCAN, which are widely used analytical XC functionals in practical calculations, were also compared. While the simple $\mathrm{NN}$ and analytical functionals demonstrated comparable accuracy, pcNN outperformed them even though the training dataset was almost the same as that of NN. Note that the mean absolute error (MAE) of pcNN-based functional is $3.6 \mathrm{kcal} / \mathrm{mol}$, which almost reaches to the error of $\operatorname{CCSD}(\mathrm{T})(\mathrm{CCSD}(\mathrm{T})$ had $3.5 \mathrm{kcal} / \mathrm{mol} \mathrm{MAE}$ for similar benchmark dataset shown in Ref. [35]).

Table III presents the benchmark results for solids, which indicate that pcNN outperformed the other XC functionals. It is remarkable that improvements for solids were achieved using only the molecular training dataset (see Appendix $\mathrm{C}$ for the difference in electron distribution between molecules and solids). For both molecules and solids, not only for the MAE, but the mean errors (ME) of NN- and pcNN-based functionals

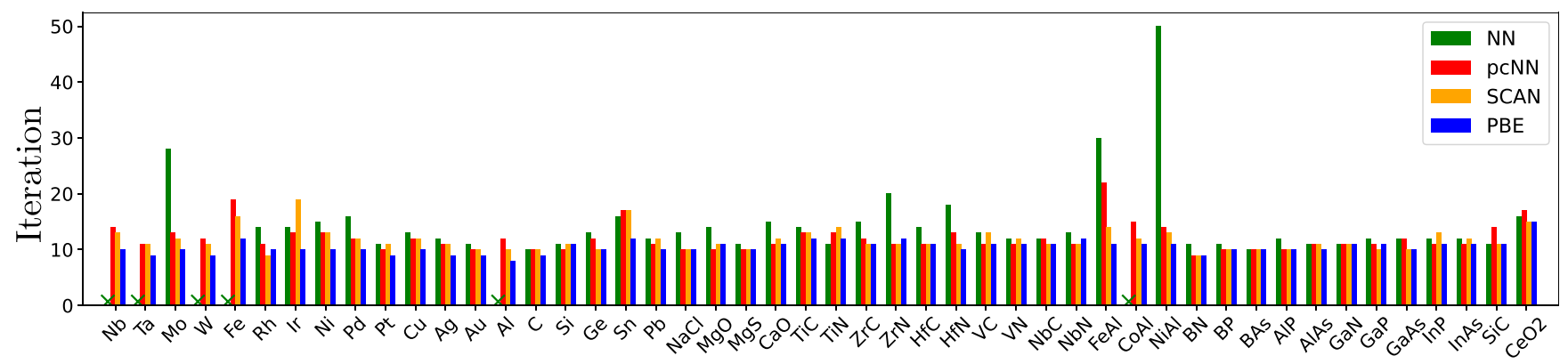

FIG. 3. Comparison of iteration number until convergence for all benchmark solids. The green $\mathrm{x}$ symbols indicate that the NN-based functional did not converge for the corresponding material. 

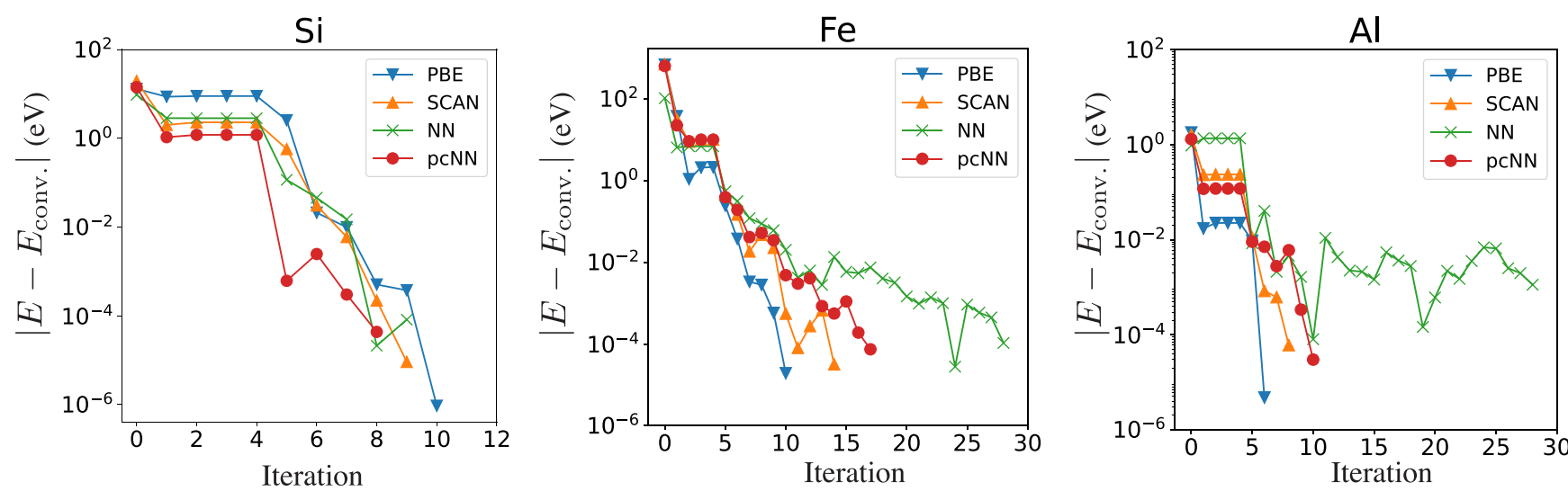

FIG. 4. Comparison of the total-energy convergence of each functional for $\mathrm{Si}, \mathrm{Al}$, and $\mathrm{Fe}$. The vertical axis displays the difference between the energy of the current step and the energy of the final iteration $E_{\text {conv. }}$. The energy value at the 30th iteration was used for $E_{\text {conv. }}$ of the $\mathrm{NN}$-based functional for $\mathrm{Al}$ as it did not converge.

were smaller than those of the analytical ones (PBE, SCAN). This indicates that the error of analytical functionals tend to have biased errors, whereas their ML-based counterparts yield random errors distributing around the exact value. Although the MAE of the NN-based functional is smaller than that of SCAN, the standard deviation (SD) of error is larger. This indicates that the $\mathrm{NN}$-based functional would yield relatively large error for some materials. The new pcNN-based functional improved in this respect as it yielded the smallest SD of error.

Figure 3 presents the number of iterations until the selfconsistent KS cycle converged. With the NN-based functional, the self-consistent calculation was not stable and did not converge for some solids. Calculation with the pcNN-based functional, in contrast, was as stable as that with analytical $\mathrm{XC}$ functionals, even though the pseudopotentials used in the calculation were adjusted for the convergence of PBE (this was also advantageous for SCAN because its structure resembles PBE). Figure 4 illustrates the process of total-energy convergence in the self-consistent $\mathrm{KS}$ cycles of $\mathrm{Si}$ (all XC functionals converged), $\mathrm{Fe}$ (NN barely converged with 30 iterations), and $\mathrm{Al}$ (NN did not converge). When the $\mathrm{KS}$ calculation successfully converged, the energy difference decreased exponentially. When $\mathrm{NN}$ failed to converge, the total energy oscillated at certain magnitudes. The convergence of pcNNbased seems much more stable than that of NN-based, and resembles SCAN, as its structure directly included SCAN. These results suggest that if a ML functional is trained as a factor to another functional, the convergence behavior will be similar.

We argue that the improvement and stability of the pcNNbased functional were due to both the training dataset and the physical constraints. As the training dataset, we used the electronic structure of small molecules whose electrons were spatially localized. In addition, some of the asymptotic constraints imposed by the $\Theta$ operation were derived from metallic solids where electrons were delocalized (e.g., constraints $\mathrm{X} 3$ and $\mathrm{C} 1$ ). Thus, the pcNN-based functional was effective for both localized and delocalized features of electrons. In the construction of the NN-based functional, the properties of the delocalized electrons were not referenced. We consider this to be the cause of the instability in the calculation of solids.

\section{B. Band structure}

Figure 5 compares band structure yielded by SCAN and pcNN XC functional. This shows that smooth band structure can be obtained both for occupied and unoccupied states with the pcNN functional. The unoccupied bands generated from pcNN tend to be shifted lower than that of SCAN.

Table IV presents experimental and calculated band gaps of some solids. We note that the band gap calculated from the KS eigenvalues does not correspond to the exact one because of differences in definitions. Regardless of the architecture, the meta-GGA functionals yield comparable results. The pcNNbased functional tends to yield slightly smaller band gaps than SCAN.

From those observations, training of ML functional referring only molecules seems to decrease the evaluated band

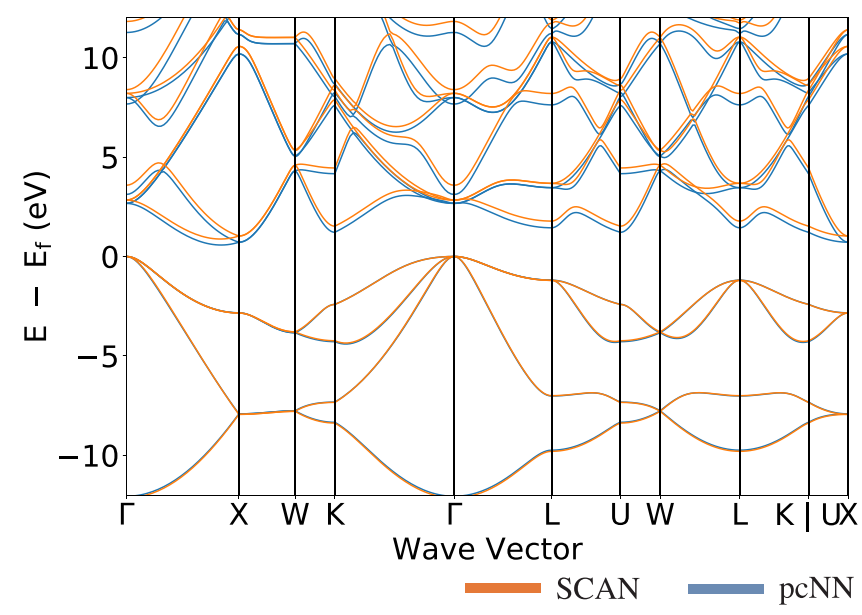

FIG. 5. Band structure of cubic diamond structure Si computed using SCAN and pcNN. 
TABLE IV. Experimental and calculated band gaps (in eV). The experimental values are cited from [37].

\begin{tabular}{lccccc}
\hline \hline & Expt. & PBE & SCAN & NN-based & pcNN-based \\
\hline $\mathrm{C}$ & 5.48 & 4.14 & 4.59 & 4.49 & 4.55 \\
$\mathrm{Si}$ & 1.17 & 0.61 & 0.83 & 0.77 & 0.80 \\
$\mathrm{InP}$ & 1.42 & 0.44 & 0.99 & 1.06 & 0.91 \\
$\mathrm{MgO}$ & 7.22 & 4.66 & 5.87 & 5.87 & 5.87 \\
$\mathrm{MgS}$ & 5.40 & 2.78 & 3.52 & 3.61 & 3.49 \\
\hline \hline
\end{tabular}

gaps. This problem should be addressed in future studies by using band structure in the training dataset. Imposing the piecewise linearity of total energy in a system with fractional electron numbers seems also effective to this problem, as Kirkpatric et al. has recently done [36]. They included training data satisfying the constraint, but it could be improved by directly imposing the constraint on the ML model as shown in this paper.

\section{Analysis of XC functional form}

To compare the form of the $\mathrm{XC}$ functionals, we plotted the $\mathrm{XC}$ energy density divided either by that in the unform electron gas limit $\varepsilon_{\mathrm{X}}^{\text {unif }}$ [3], namely the enhancement factor, or by that of SCAN $\varepsilon_{\mathrm{XC}}^{\mathrm{SCAN}}$ (Fig. 6). Because of the imposed physical constraints, pcNN is identical to SCAN and PBE at the uniform electron gas conditions. The difference between pcNN and SCAN functionals is $\sim 2 \%$, whereas they provide much more different results as shown in II-III. The difference in XC energy density is largest around $s \sim 1$ and $\tau / \tau_{\text {unif }} \sim 0.5$. Considering the density distribution of the training molecules (Fig. 7), the electron around $r_{s} \sim 1$ contributed significantly to the training. Note that the density around $r_{s} \sim 1$ is lower than that in the core region but higher than that at the edge of valence region.

\section{CONCLUSIONS}

In this paper, we proposed a method to analytically impose asymptotic constraints on ML models, and applied it to construct the XC functional of DFT. Our XC functional based on the proposed NN model satisfied physical asymptotic constraints. As a result, the self-consistent solution of the KS equation converged stably and exhibited higher accuracy than existing standard $\mathrm{XC}$ functionals, even for solid systems that were not included in the training dataset. This improvement can be attributed to both the flexibility of the ML model and the regularization by imposing physical constraints. By incorporating these advantages, the performance of the constructed $\mathrm{XC}$ functional was improved.
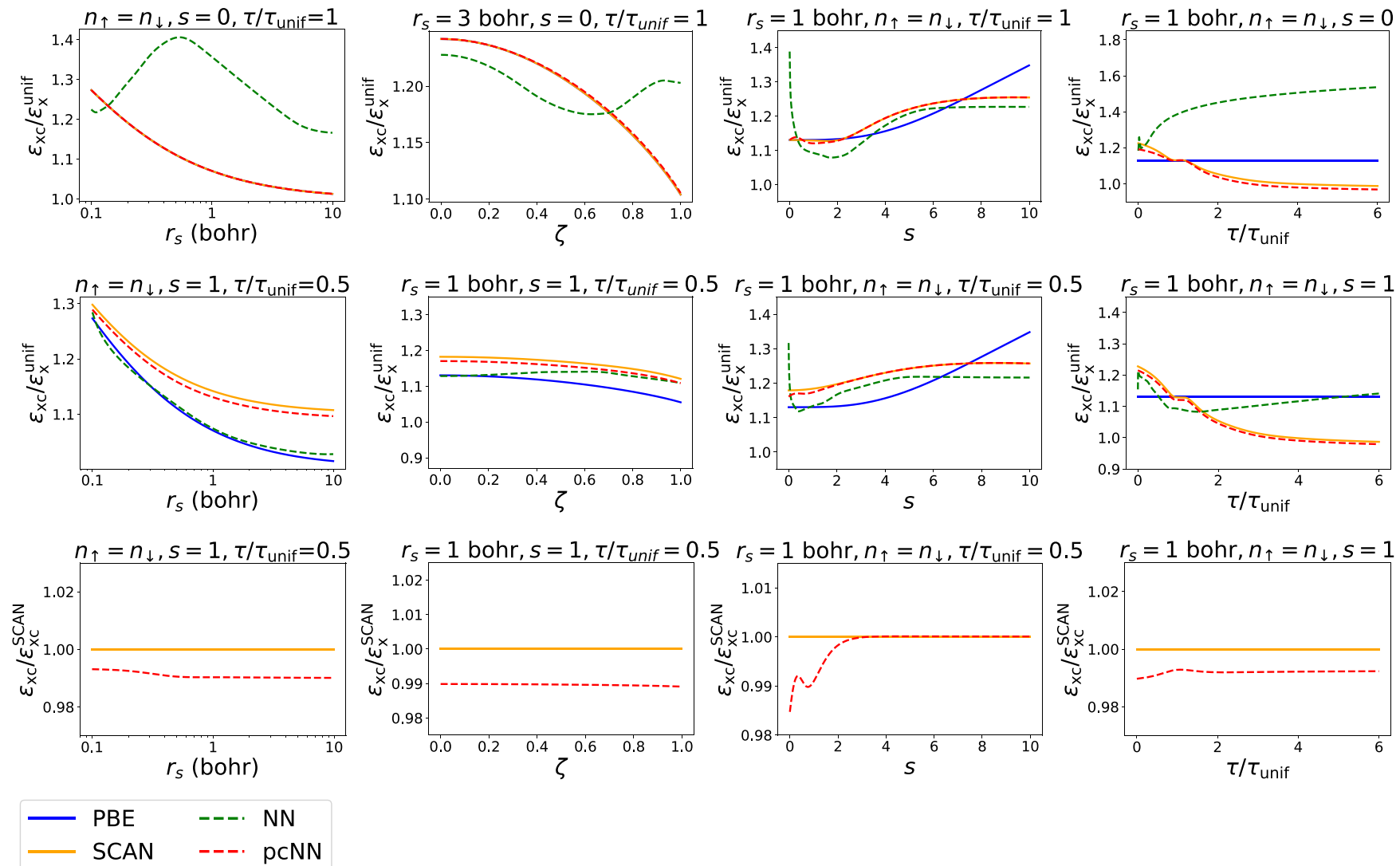

FIG. 6. Plots of the XC functional forms of PBE, SCAN, NN-based, and pcNN-based functional along with the four descriptors of metaGGA in Eq. (10). The top row exhibits the enhancement factor around the uniform electron condition, while the middle row exhibits the enhancement factor in non-uniform situations. The bottom row compares the difference between the pcNN and SCAN functional by plotting the factor from SCAN. 
$\mathrm{XC}$ functionals that have been widely used in application studies, such as PBE and SCAN, are stable and accurate for a variety of materials. One of their common features is that they satisfy many analytic physical constraints. Similarly, pcNN is designed to satisfy as many physical constraints as possible analytically. Its high stability and accuracy are expected to be useful in practical research in the future.

The ML modeling method presented in this paper is not limited to the construction of XC functionals, but will also be applicable to other ML applications where theoretical asymptotic constraints are known.

\section{ACKNOWLEDGMENTS}

This work was supported by KAKENHI Grant No. JP20J20845 from Japan Society for the Promotion of Science. Part of the calculation was performed at the Supercomputer System B and C at the Institute for Solid State Physics, the University of Tokyo.

\section{APPENDIX A: ACTIVATION FUNCTIONS}

Since the variable range of the output value $F$ is $[0, \infty)$, that of the activation function should also be semi-infinite. Additionally, it is desirable for the function to be infinitely differentiable because its derivative is substituted in the KS equation [see Eq. (7)]. Considering these requirements, we adopted the softplus [38] as the activation function $\phi(\mathbf{x})$ and changed its coefficients as follows to satisfy $\phi(0)=1$ and $\phi^{\prime}(0)=1$ :

$$
\phi(\mathbf{x})=\frac{1}{\log 2} \log (1+\exp (2 \mathbf{x} \log 2)) .
$$

With these adjustments, when all NN weights are set to $0, F_{\mathrm{X}, \mathrm{C}}^{\mathrm{NN}}$ converges to 1 , and the total $\mathrm{XC}$ functional thus converges to SCAN.
Since $F_{\mathrm{X}}$ and $F_{\mathrm{C}}$ should be non-negative (see constraints $\mathrm{X} 4$ and $\mathrm{C} 5$ in Table I), these activation functions were also applied after each $\Theta$ operation (see Fig. 1):

$$
F=\phi\left(F_{\mathrm{X}, \mathrm{C}}^{\mathrm{tot}}-1\right) \text {. }
$$

\section{APPENDIX B: TRAINING PROCEDURES}

The loss function in Eq. (17) is computed by solving the KS equation [Eq. (3)] with substituting the NN-based XC functional during training. The KS equation is a self-consistent eigenvalue equation, and thus it is technically difficult to calculate its derivative. In this study, to avoid using gradients, we performed simulated-annealing-based optimization $[39,40]$. We denote all the weights of the matrices and vectors in $\mathrm{NN}$ as $\mathbf{w} . \mathbf{w}$ was randomly perturbated in every trial step. If $\mathrm{NN}$ improved the loss value, the perturbation was accepted; otherwise, it was rejected. We set the step size of the random number generation to $\sigma=0.003$ and the imaginary temperature to $T=0.1 \Delta_{i}^{t-1}$.

This training was performed with 2560 parallel threads and approximately 300 trial steps for updating per thread on a supercomputer system with AMD EPYC 7702 and 2 GB RAM per core. The overall training procedure in each parallel thread is described in Algorithm 1. After this iteration, we collected the trained weights $\mathbf{w}_{i}^{t}$ and loss values $\Delta_{i}^{t}$ from all the parallel threads and trial steps and sorted them by $\Delta_{i}^{t}$. For the top 100 weights, we computed the mean absolute error for the dataset consisting of the AE of 144 molecules. The molecules were small to medium-sized (the largest molecule was benzene), whose accurate WFT data are available [26]. Finally, the weights with the highest accuracy were adopted. The computational cost of this training was high because the KS equation for each material was solved in each iteration. Recently, Li et al. [41], Kasim et al. [35], and Dick et al. [42] implemented back propagation of the KS equation for some systems to perform gradient-based optimization. Although we

Algorithm 1 Pipeline of training the $\mathrm{NN}$ in a parallel thread $i$.

Parameter: Step size $\sigma$, imaginary temperature $T$, and number of iterations $N_{\text {iter. }}$.

1: $\quad$ Let $\mathbf{w}_{i}^{0}=\mathbf{0}$ and $\Delta_{i}^{0}=\infty$.

2: Generate random-weight-perturbation $\delta \mathbf{w}_{i}^{0}$ from $\mathcal{N}(0, \sigma)$, where $\mathcal{N}(a, b)$ represents the normal distribution with mean $a$ and standard deviation $b$.

3: for $t=1$ to $N_{\text {iter. }}$ do

4: Substitute the NN-based XC functional with parameter $\mathbf{w}_{i}^{t-1}+\delta \mathbf{w}_{i}^{t-1}$ into the KS equation and solve it for the energies and densities to calculate the loss value $\Delta_{i}^{t}$ by Eq. (17).

5: Compute $P=\exp \left(-\left(\Delta_{i}^{t}-\Delta_{i}^{t-1}\right) / T\right)$.

6: Generate random number $p_{i}^{t} \in(0,1)$ from a uniform distribution.

7: if $P>1$ then

8: $\quad$ Set $\mathbf{w}_{i}^{t}=\mathbf{w}_{i}^{t-1}+\delta \mathbf{w}_{i}^{t-1}$ and $\delta \mathbf{w}_{i}^{t}=\delta \mathbf{w}_{i}^{t-1}$.

9: else if $p<P<1$ then

10: Set $\mathbf{w}_{i}^{t}=\mathbf{w}_{i}^{t-1}+\delta \mathbf{w}_{i}^{t-1}$ and generate the random weight perturbation $\delta \mathbf{w}_{i}^{t}$ from $\mathcal{N}(0, \sigma)$.

11: else

12: Set $\mathbf{w}_{i}^{t}=\mathbf{w}_{i}^{t-1}$ and $\Delta_{i}^{t}=\Delta_{i}^{t-1}$, and generate the random weight perturbation $\delta \mathbf{w}_{i}^{t}$ from $\mathcal{N}(0, \sigma)$.

13: end if

14: end for 
avoided using these methods owing to the lack of memory in our environment, they have the potential to improve the training efficiency. Directly referring the XC energy density obtained from the Wu-Yang KS inversion method [43-45] would also be efficient.

\section{APPENDIX C: DIFFERENCE IN DENSITY DISTRIBUTION BETWEEN MOLECULES AND SOLIDS}

Figure 7 presents the difference of density distribution between molecules (ones used in the training: $\mathrm{H}_{2} \mathrm{O}, \mathrm{NH}_{3}$, and $\mathrm{CH}_{2}$ on the PySCF grids) and solids ( $\mathrm{Si}, \mathrm{Fe}$, and $\mathrm{Al}$, on the VASP grids) by plotting the local Wigner-Seitz radius of electrons $r_{s}$ vs dimensionless density gradient $s$ and $\tau / \tau_{\text {unif }}$. The difference in trends of density distribution between molecules and solids came from the property of basis function used in each program (Gaussian and plane-wave basis). The density distributions of molecules and solids were similar around $r_{s} \sim 1$, while they were quite different in other regions. The physical constraints imposed to pcNN seems to have suppressed unreliable ML output in such extrapolate density region.
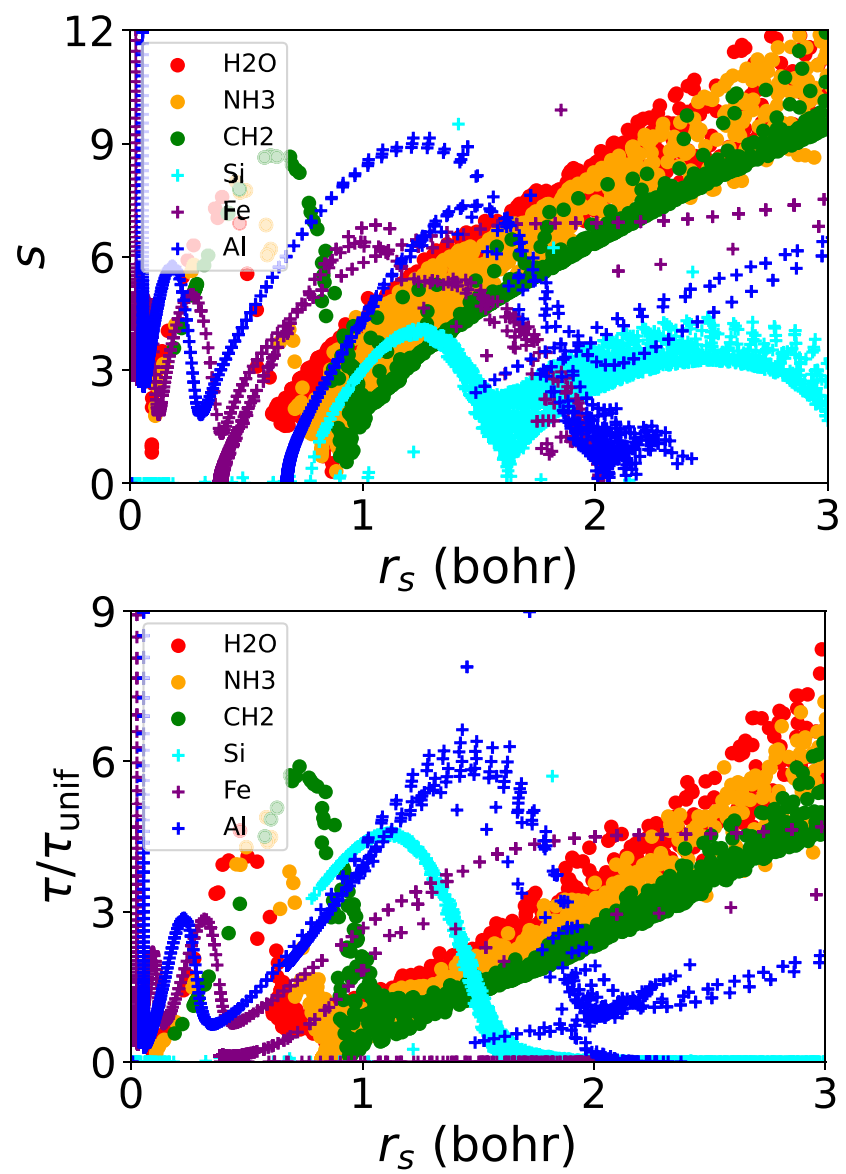

FIG. 7. Density distribution of molecules and solids, $r_{s}$ vs $s$ (upper) and $r_{s}$ vs $\tau / \tau_{\text {unif }}$ (lower). The o symbols indicate density distribution of molecules used in the training of pcNN-based functional, while the + symbols indicate density distribution of solids.

\section{APPENDIX D: DEPENDENCE OF LEARNING EFFICIENCY ON TRAINING DATASET}

To investigate how the performance of the $\mathrm{XC}$ functional depends on the molecules referred to in the training, we trained the pcNN functionals using four different datasets. Dataset 1 includes only atomization energy and density of $\mathrm{H}_{2} \mathrm{O}$ and $\mathrm{NH}_{3}$ with the following loss function:

$$
\Delta=c_{1}\left(\Delta_{\mathrm{AE}}^{\mathrm{H}_{2} \mathrm{O}}+\Delta_{\mathrm{AE}}^{\mathrm{NH}_{3}}\right) / \mathrm{AE}_{0}+c_{3}\left(\Delta_{n}^{\mathrm{H}_{2} \mathrm{O}}+\Delta_{n}^{\mathrm{NH}_{3}}\right) .
$$

Dataset 2 is an extension of dataset 1 with the atomization energy and density of $\mathrm{CH}_{2}$, a radical with spin polarization:

$$
\begin{aligned}
\Delta= & c_{1}\left(\Delta_{\mathrm{AE}}^{\mathrm{H}_{2} \mathrm{O}}+\Delta_{\mathrm{AE}}^{\mathrm{NH}_{3}}+\Delta_{\mathrm{AE}}^{\mathrm{CH}_{2}}\right) / \mathrm{AE}_{0} \\
& +c_{3}\left(\Delta_{n}^{\mathrm{H}_{2} \mathrm{O}}+\Delta_{n}^{\mathrm{NH}_{3}}+\Delta_{n}^{\mathrm{CH}_{2}}\right) .
\end{aligned}
$$

Dataset 3 is an extension of dataset 2 with the ionization energy of $\mathrm{H}_{2} \mathrm{O}$ :

$$
\begin{aligned}
\Delta= & c_{1}\left(\Delta_{\mathrm{AE}}^{\mathrm{H}_{2} \mathrm{O}}+\Delta_{\mathrm{AE}}^{\mathrm{NH}_{3}}+\Delta_{\mathrm{AE}}^{\mathrm{CH}_{2}}\right) / \mathrm{AE}_{0}+c_{2} \Delta_{\mathrm{IP}}^{\mathrm{H}_{2} \mathrm{O}} / \mathrm{IP}_{0} \\
& +c_{3}\left(\Delta_{n}^{\mathrm{H}_{2} \mathrm{O}}+\Delta_{n}^{\mathrm{NH}_{3}}+\Delta_{n}^{\mathrm{CH}_{2}}\right) .
\end{aligned}
$$

Dataset 4 is an extension of dataset 3 with the ionization energy of $\mathrm{NH}_{3}$ and $\mathrm{CH}_{2}$, which corresponds to the dataset demonstrated in the main text:

$$
\begin{aligned}
\Delta= & c_{1}\left(\Delta_{\mathrm{AE}}^{\mathrm{H}_{2} \mathrm{O}}+\Delta_{\mathrm{AE}}^{\mathrm{NH}_{3}}+\Delta_{\mathrm{AE}}^{\mathrm{CH}_{2}}\right) / \mathrm{AE}_{0} \\
& +c_{2}\left(\Delta_{\mathrm{IP}}^{\mathrm{H}_{2} \mathrm{O}}+\Delta_{\mathrm{IP}}^{\mathrm{NH}_{3}}+\Delta_{\mathrm{IP}}^{\mathrm{CH}_{2}}\right) / \mathrm{IP}_{0} \\
& +c_{3}\left(\Delta_{n}^{\mathrm{H}_{2} \mathrm{O}}+\Delta_{n}^{\mathrm{NH}_{3}}+\Delta_{n}^{\mathrm{CH}_{2}}\right) .
\end{aligned}
$$

Figure 8 exhibits how the performance changed as the training progressed. At every 50 training steps, we took the weights with the smallest loss function value from the parallel threads and compared their performance for the molecular benchmark dataset.

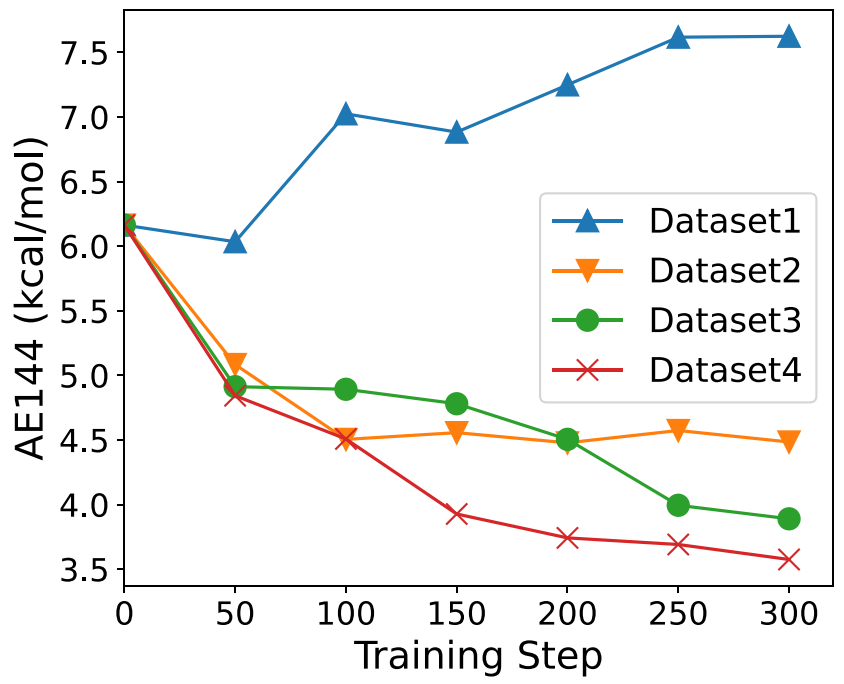

FIG. 8. Transition of performances of pcNN functionals trained with different datasets. The definition of each dataset is described in Eq. (D1)-(D4). "AE144" represents the mean absolute error for 144 molecules, which corresponds to the definition described in the main text, Sec. II F. 
Dataset 3, which included ionization energy in addition to dataset 2, improved the performance for atomization energy, though it slowed the convergence. The training using dataset 4 , which included additional ionization energy, resulted in a trivial improvement in accuracy, whereas it improved the convergence speed. From this investigation we found that adding qualitatively different data (dataset $1 \rightarrow 2$ and $2 \rightarrow 3$ ) tends to improve the accuracy, while adding of the same type of data (dataset $3 \rightarrow 4$ ) tends to accelerate the learning speed.
[1] P. Hohenberg and W. Kohn, Inhomogeneous electron gas, Phys. Rev. 136, B864 (1964).

[2] W. Kohn and L. J. Sham, Self-consistent equations including exchange and correlation effects, Phys. Rev. 140, A1133 (1965).

[3] J. C. Slater, A simplification of the Hartree-Fock method, Phys. Rev. 81, 385 (1951).

[4] M. G. Medvedev, I. S. Bushmarinov, J. Sun, J. P. Perdew, and K. A. Lyssenko, Density functional theory is straying from the path toward the exact functional, Science 355, 49 (2017).

[5] J. C. Snyder, M. Rupp, K. Hansen, K.-R. Müller, and K. Burke, Finding Density Functionals with Machine Learning, Phys. Rev. Lett. 108, 253002 (2012).

[6] F. Brockherde, L. Vogt, L. Li, M. E. Tuckerman, K. Burke, and K.-R. Müller, Bypassing the Kohn-Sham equations with machine learning, Nat. Commun. 8, 872 (2017).

[7] R. Nagai, R. Akashi, S. Sasaki, and S. Tsuneyuki, Neuralnetwork Kohn-Sham exchange-correlation potential and its out-of-training transferability, J. Chem. Phys. 148, 241737 (2018).

[8] R. Nagai, R. Akashi, and O. Sugino, Completing density functional theory by machine learning hidden messages from molecules, npj Comput. Mater. 6, 43 (2020).

[9] J. Hollingsworth, L. Li, T. E. Baker, and K. Burke, Can exact conditions improve machine-learned density functionals?, J. Chem. Phys. 148, 241743 (2018).

[10] S. H. Vosko, L. Wilk, and M. Nusair, Accurate spin-dependent electron liquid correlation energies for local spin density calculations: A critical analysis, Can. J. Phys. 58, 1200 (1980).

[11] J. P. Perdew, K. Burke, and M. Ernzerhof, Generalized Gradient Approximation Made Simple, Phys. Rev. Lett. 77, 3865 (1996).

[12] J. Tao, J. P. Perdew, V. N. Staroverov, and G. E. Scuseria, Climbing the Density Functional Ladder: Nonempirical MetaGeneralized Gradient Approximation Designed for Molecules and Solids, Phys. Rev. Lett. 91, 146401 (2003).

[13] J. Sun, A. Ruzsinszky, and J. P. Perdew, Strongly Constrained and Appropriately Normed Semilocal Density Functional, Phys. Rev. Lett. 115, 036402 (2015).

[14] Y. Zhao and D. G. Truhlar, The M06 suite of density functionals for main group thermochemistry, thermochemical kinetics, noncovalent interactions, excited states, and transition elements: Two new functionals and systematic testing of four M06-class functionals and 12 other functionals, Theor. Chem. Acc. 120, 215 (2008).

[15] M. Levy and J. P. Perdew, Hellmann-Feynman, virial, and scaling requisites for the exact universal density functionals. Shape of the correlation potential and diamagnetic susceptibility for atoms, Phys. Rev. A 32, 2010 (1985).

[16] G. L. Oliver and J. P. Perdew, Spin-density gradient expansion for the kinetic energy, Phys. Rev. A 20, 397 (1979).
[17] J. Bradbury, R. Frostig, P. Hawkins, M. J. Johnson, C. Leary, D. Maclaurin, G. Necula, A. Paszke, J. VanderPlas, S. WandermanMilne, and Q. Zhang, JAX: Composable transformations of Python+NumPy programs (2018), https://github.com/google/ jax.

[18] J. P. Perdew, A. Ruzsinszky, J. Sun, and K. Burke, Gedanken densities and exact constraints in density functional theory, J. Chem. Phys. 140, 18A533 (2014).

[19] J. P. Perdew and Y. Wang, Accurate and simple analytic representation of the electron-gas correlation energy, Phys. Rev. B 45, 13244 (1992).

[20] M. Levy, Density-functional exchange correlation through coordinate scaling in adiabatic connection and correlation hole, Phys. Rev. A 43, 4637 (1991).

[21] G. D. Purvis and R. J. Bartlett, A full coupled-cluster singles and doubles model: The inclusion of disconnected triples, J. Chem. Phys. 76, 1910 (1982).

[22] Q. Sun, T. C. Berkelbach, N. S. Blunt, G. H. Booth, S. Guo, Z. Li, J. Liu, J. D. McClain, E. R. Sayfutyarova, S. Sharma, S. Wouters, and G. K. Chan, PySCF: The python-based simulations of chemistry framework, Wiley Interdiscip. Rev. Comput. Mol. Sci. 8, e1340 (2018).

[23] https://github.com/ml-electron-project/pcNN_mol.

[24] https://github.com/ml-electron-project/pcNN_solid.

[25] https://github.com/ml-electron-project/pcNN_train.

[26] L. A. Curtiss, K. Raghavachari, P. C. Redfern, and J. A. Pople, Assessment of Gaussian-2 and density functional theories for the computation of enthalpies of formation, J. Chem. Phys. 106, 1063 (1997).

[27] P. Haas, F. Tran, and P. Blaha, Calculation of the lattice constant of solids with semilocal functionals, Phys. Rev. B 79, 085104 (2009).

[28] G. Kresse and J. Furthmüller, Efficiency of ab-initio total energy calculations for metals and semiconductors using a plane-wave basis set, Comput. Mat. Sci. 6, 15 (1996).

[29] G. Kresse and J. Furthmüller, Efficient iterative schemes for ab initio total-energy calculations using a plane-wave basis set, Phys. Rev. B 54, 11169 (1996).

[30] G. Kresse and D. Joubert, From ultrasoft pseudopotentials to the projector augmented-wave method, Phys. Rev. B 59, 1758 (1999).

[31] P. E. Blöchl, Projector augmented-wave method, Phys. Rev. B 50, 17953 (1994).

[32] P. Pulay, Improved SCF convergence acceleration, J. Comput. Chem. 3, 556 (1982).

[33] P. Pulay, Convergence acceleration of iterative sequences. The case of SCF iteration, Chem. Phys. Lett. 73, 393 (1980).

[34] See Supplemental Material at http://link.aps.org/supplemental/ 10.1103/PhysRevResearch.4.013106 for individual errors of molecules and solids included in the benchmark dataset. 
[35] M. F. Kasim and S. M. Vinko, Learning the ExchangeCorrelation Functional from Nature with Fully Differentiable Density Functional Theory, Phys. Rev. Lett. 127, 126403 (2021).

[36] J. Kirkpatrick, B. McMorrow, D. H. Turban, A. L. Gaunt, J. S. Spencer, A. G. Matthews, A. Obika, L. Thiry, M. Fortunato, D. Pfau et al., Pushing the frontiers of density functionals by solving the fractional electron problem, Science 374, 1385 (2021).

[37] J. Heyd, J. E. Peralta, G. E. Scuseria, and R. L. Martin, Energy band gaps and lattice parameters evaluated with the HeydScuseria-Ernzerhof screened hybrid functional, J. Chem. Phys. 123, 174101 (2005).

[38] C. Dugas, Y. Bengio, F. Bélisle, C. Nadeau, and R. Garcia, Incorporating second-order functional knowledge for better option pricing, in Advances in Neural Information Processing Systems 13 (NIPS, 2000), p. 472.

[39] S. Kirkpatrick, C. D. Gelatt, and M. P. Vecchi, Optimization by simulated annealing, Science 220, 671 (1983).
[40] V. Černý, Thermodynamical approach to the traveling salesman problem: An efficient simulation algorithm, J. Optim. Theory Appl. 45, 41 (1985).

[41] L. Li, S. Hoyer, R. Pederson, R. Sun, E. D. Cubuk, P. Riley, and K. Burke, Kohn-Sham Equations as Regularizer: Building Prior Knowledge into Machine-Learned Physics, Phys. Rev. Lett. 126, 036401 (2021).

[42] S. Dick and M. Fernandez-Serra, Highly accurate and constrained density functional obtained with differentiable programming, Phys. Rev. B 104, L161109 (2021).

[43] Q. Zhao, R. C. Morrison, and R. G. Parr, From electron densities to Kohn-Sham kinetic energies, orbital energies, exchange-correlation potentials, and exchange-correlation energies, Phys. Rev. A 50, 2138 (1994).

[44] Q. Wu and W. Yang, A direct optimization method for calculating density functionals and exchange-correlation potentials from electron densities, J. Chem. Phys. 118, 2498 (2003).

[45] S. Nam, R. J. McCarty, H. Park, and E. Sim, KS-pies: KohnSham inversion toolkit, J. Chem. Phys. 154, 124122 (2021). 